\title{
Non-Equilibrium Dynamics of One-dimensional Infinite Particle Systems with a Hard-Core Interaction
}

\author{
R. L. Dobrushin ${ }^{1}$ and J. Fritz ${ }^{2}$ \\ ${ }^{1}$ Institute for Problems of Information Transmission, SU-111024, Moscow E-24, USSR \\ ${ }^{2}$ Mathematical Institute, Budapest, Hungary
}

\begin{abstract}
An infinite system of Newton's equation of motion is considered for one-dimensional particles interacting by a finite-range hard-core potential of singularity like an inverse power of distance between the hard cores. Existence of limiting solutions is proved for initial configurations of finite specific energy and the semigroup of motion is constructed if energy fluctuations near infinity increase only as a small power of distance from the origin. In this case uniqueness of solutions is also proved and the solution is a weakly continuous function of initial data. The allowed set of initial configurations carries a wide class of probability measures including Gibbsian fields with different potentials. In the absence of hard cores limiting solutions are constructed for initial configurations with a logarithmic order of energy and density fluctuations.
\end{abstract}

\section{Introduction}

The aim of this paper is to extend the results by Harris [1] and Lanford [2] on the existence of non-equilibrium dynamics of infinitely many particles on the line. The interaction is given by a translation invariant hard-core pair potential $U$ of finite range, thus the interparticle force is just the negative derivative of $U$ and the equations of motion are those of classical mechanics with this conservative force. As it is well known (see $[1,2,5]$ ) a solution to such an infinite system of differential equations exists only for a relatively small set of initial configurations, and the solution, if any, is not unique in the usual sense.

For the purposes of non-equilibrium statistical mechanics the semigroup of motion should be constructed in a set of configurations large enough to carry a class of Gibbsian fields. Let us remark that equilibrium dynamics that is a semigroup acting in the support of a Gibbsian field associated with the same potential as the dynamics itself has been constructed by several authors ([3-7]) while Lang [8] considers a first order system with additional white-noise terms. As regards nonequilibrium dynamics, in Harris' collision model (zero-range interaction) $U$ is formally the $\delta$-function, Lanford [2] treates the case of not necessarily symmetric, 
smooth interactions and proves existence of solutions for initial configurations of a logarithmic order of velocity and density fluctuations.

In this paper the main condition on the interaction potential is that its singularity near the hard core is not stronger than that of an inverse power at 0 . Solutions associated with initial configurations of finite specific energy are constructed as weak limits of solutions to finite subsystems. Assuming that $U$ is repulsive enough we show that the equations of motion satisfy a quasi-Lipschitz condition; hence we deduce that solutions form a reversible semigroup e.g. in the set $\bar{\Omega}_{0}$ of configurations with a logarithmic order of energy fluctuation, and the motion is a uniquely determined weakly continuous function of its initial data. $\bar{\Omega}_{0}$ is of full measure with respect to any Gibbsian field with potential $\tilde{U}$ such that the singularity of $\tilde{U}$ is not weaker than that of $U$. In the absence of hard cores solutions are constructed in the subset $\Omega_{0}$ of $\bar{\Omega}_{0}$ characterized by a logarithmic order of density fluctuation. In the proofs the conservative nature of interparticle forces is essentially exploited, the a priori bound implying compactness of the sequence of solutions to finite subsystems is deduced from the law of energy conservation. Such ideas will be applied to prove two-dimensional existence theorems in a forthcoming paper [9] by the authors.

\section{Preliminaries}

First we specify notation and terminology used throughout this paper. In general, $x_{i}$ and $v_{i}, i \in I$ denote the position and the velocity of the $i$-th particle; $I$ is the set of integers, $x_{i}$ and $v_{i}$ are real numbers. Let $\Omega$ denote the set of all locally finite labelled configurations satisfying the hard-core condition $\left|x_{i}-x_{j}\right|>\delta$ if $i \neq j ; \delta \geqq 0$ will be the hard-core diameter of our potential $U$. We may and do assume that particles are numbered in an increasing order of positions so that a configuration $\omega \in \Omega$ is a doubly infinite sequence $\omega=\left\{\left(x_{i}, v_{i}\right) ; i \in I\right\}$ of pairs of real numbers such that $x_{i+1}$ $>x_{i}+\delta$. In the special case of $\delta=0$ we need the condition of local finiteness as well, i.e. only such configurations are considered where the sequence of positions has no limit points. Configurations differing only in the way of enumeration of particles are usually identified, but the equations of motion and some quantities will be formulated in terms of labelled configurations. If necessary, the position and the velocity of the $i$-th particle in $\omega$ will be denoted as $x_{i}=x_{i}(\omega)$ and $v_{i}=v_{i}(\omega)$, respectively. The configuration space $\Omega$ is equipped with the weak topology, i.e. $\lim \omega_{n}=\omega$ means that $\lim _{n} x_{i}\left(\omega_{n}\right)=x_{i}(\omega)$ and $\lim _{n} v_{i}\left(\omega_{n}\right)=v_{i}(\omega)$ for each $i$.

Trajectories in $\Omega$ are always parametrized by the time $t$ from 0 to $+\infty$, the set of weakly continuous trajectories $\omega_{t}=\varphi(t), \omega_{t} \in \Omega$ for $t \geqq 0$ will be denoted by $\Omega[0, \infty)$. Due to the continuity of individual trajectories $x_{i}\left(\omega_{t}\right), v_{i}\left(\omega_{t}\right)$, particles along a trajectory $\omega_{t} \in \Omega[0, \infty)$ preserve their initial numbering. Convergence $\lim \omega_{t}^{n}=\omega_{t}$ in $\Omega[0, \infty)$ is defined by

$$
\lim _{n} \sup _{0 \leqq t \leqq T}\left[\left|x_{i}\left(\omega_{t}^{n}\right)-x_{i}\left(\omega_{t}\right)\right|+\left|v_{i}\left(\omega_{t}^{n}\right)-v_{i}\left(\omega_{t}\right)\right|\right]=0
$$

for each $T>0$ and $i \in I$ without any uniformity assumption. A family $\omega_{t}=\varphi(t, \omega)$, $\omega \in \Omega^{\prime}, t \geqq 0$ of weakly continuous trajectories is a reversible semigroup in $\Omega^{\prime} \subset \Omega$ if 
$\omega_{t} \in \Omega^{\prime}$ for each $t, \varphi(0, \omega)=\omega$ and $\varphi(t+s, \omega)=\varphi(t, \varphi(s, \omega)), \varphi\left(t,[\varphi(t, \omega)]^{+}\right)=\omega^{+}$, $t, s \geqq 0$ are identities; in the second one ${ }^{+}$indicates that the velocities have been reversed, i.e. $x_{i}\left(\omega^{+}\right)=x_{i}(\omega), v_{i}\left(\omega^{+}\right)=-v_{i}(\omega)$ for each $i$.

Suppose now that we are given a translation invariant symmetric pair potential $U$ of hard-core diameter $\delta \geqq 0$ and range $R>\delta$ that is an even function $U=U(x)$, $-\infty<x<+\infty$, such that $U(x)=+\infty$ if $|x| \leqq \delta,|U(x)|<+\infty$ if $|x|>\delta, U(x)=0$ if $|x|>R$, further $U$ is continuously differentiable for $|x|>\delta$ and $\lim U(x)=+\infty$ if $x$ approaches $\delta$. For convenience we assume that the derivative $U^{\prime}$ of $U$ satisfies a local Lipschitz condition for $|x|>\delta$ that means finiteness of

$$
L(u)=\sup _{x, y}\left\{\left|\frac{U^{\prime}(x)-U^{\prime}(y)}{x-y}\right| ; \quad U(x) \leqq u, U(y) \leqq u, x \neq y\right\}
$$

for $u<+\infty$. A weakly continuous trajectory $\omega_{t} \in \Omega[0, \infty)$ is called a (global) solution to the equations of motion with initial condition $\omega \in \Omega$ if $\omega_{0}=\omega$, the individual trajectories $x_{i}=x_{i}\left(\omega_{t}\right), v_{i}=v_{i}\left(\omega_{t}\right), t \geqq 0$ are differentiable and satisfy the equations of motion

$$
\frac{d v_{i}}{d t}=-\sum_{j \neq i} U^{\prime}\left(x_{i}-x_{j}\right), \quad \frac{d x_{i}}{d t}=v_{i} ; \quad i \in I
$$

for $t \geqq 0$. We have assumed here that particles are of unit mass. Let us remark that the sum in (1) is always finite since only locally finite configurations are considered.

Owing to the law of energy conservation the initial value problem has a unique solution to any finite subsystem of (1), thus we have a possibility to define a sequence $\varphi_{n}(t, \omega) \in \Omega[0, \omega), n=1,2, \ldots$ of approximate solutions for each initial configuration $\omega \in \Omega$, e.g. as follows. Consider the $2 n+1$ pairs of equations from (1) associated with particles numbered by $i,|i| \leqq n$ and let $\left\{x_{i}(t), v_{i}(t) ;|i| \leqq n\right\}$ denote the solution of this system with initial data $x_{i}(0)=x_{i}(\omega), v_{i}(0)=v_{i}(\omega), \omega \in \Omega$; then $\varphi_{n}$ is defined by

$$
\begin{aligned}
& x_{i}\left(\varphi_{n}\right)=x_{i}(t), \quad v_{i}\left(\varphi_{n}\right)=v_{i}(t) \quad \text { if } \quad|i| \leqq n, \\
& x_{i}\left(\varphi_{n}\right)=x_{n}(t)+(i-n) R \quad \text { if } i>n, \\
& x_{i}\left(\varphi_{n}\right)=x_{-n}(t)+(i+n) R \quad \text { if } \quad i<-n, \\
& v_{i}\left(\varphi_{n}\right)=0 \text { if } \quad|i|>n .
\end{aligned}
$$

It is easy to check that $x_{i}\left(\varphi_{n}\right)$ and $\frac{d}{d t} x_{i}\left(\varphi_{n}\right)$ satisfy the equations of motion for each $i$, further $\varphi_{n}(t+s, \omega)=\varphi_{n}\left(t, \varphi_{n}(s, \omega)\right), \varphi_{n}\left(t,\left[\varphi_{n}(t, \omega)\right]^{+}\right)=\varphi_{n}\left(0, \omega^{+}\right)$if $t, s \geqq 0$.

A solution $\omega_{t}$ is called a limiting solution if we can select a subsequence $n_{k}$ such that $\lim _{k} \varphi_{n_{k}}\left(t, \omega_{0}\right)=\omega_{t}$ in the topology of $\Omega[0, \infty)$. To prove existence of a limiting solution with initial configuration $\omega$, compactness of the sequence of approximate solutions is needed that means a bound $\left|v_{i}\left(\varphi_{n}(t, \omega)\right)\right| \leqq q_{i}(t, \omega)$ such that $q_{i}$ does not depend on $n$ and it is bounded in finite intervals of time. The basic problem is to find a large enough set in $\Omega$ where such an a priori bound holds. 


\section{Main Result}

Integrals of motion as total energy and particle number play a decisive role in the study of system (1). Let $f_{0}=f_{0}(x, \sigma)$ denote the indicator of $[-\sigma, \sigma], \sigma>0$, i.e. $f_{0}=1$ if $|x| \leqq \sigma$ and $f_{0}=0$ otherwise, further $x_{i}=x_{i}(\omega), v_{i}=v_{i}(\omega), \omega \in \Omega$; then

$$
N(\omega, \mu, \sigma)=\sum_{i \in I} f_{0}\left(x_{i}-\mu, \sigma\right)
$$

and

$$
H(\omega, \mu, \sigma)=\sum_{i \in I} f_{0}\left(x_{i}-\mu, \sigma\right) \frac{1}{2}\left[v_{i}^{2}+\sum_{j \neq i} f_{0}\left(x_{j}-\mu, \sigma\right) U\left(x_{i}-x_{j}\right)\right]
$$

are the particle number and the total energy of $\omega$ in the interval $[\mu-\sigma, \mu+\sigma]$.

An essential difference between the special cases $\delta>0$ and $\delta=0$ is that the number of nonzero summands in $U_{i}=\sum_{j \neq i} U\left(x_{i}-x_{j}\right)$ can't exceed $2 \frac{R}{\delta}$ if $\delta>0$ while it may be arbitrarily large if $\delta=0$. From now on we assume that $\delta>0$, the case $\delta=0$ will be discussed in Section 8 .

Since $\min _{x} U(x) \geqq-\frac{a}{b}$ with $a>0, b>0$ as specified in condition (E) below, any partial sum $V_{i}$ of $U_{i}$ satisfies $V_{i} \geqq-2 \frac{a R}{b \delta}$, thus putting $B=\frac{a R}{b \delta}$ we obtain a nonnegative version

$$
Q(\omega, \mu, \sigma)=B N(\omega, \mu, \sigma)+H(\omega, \mu, \sigma)
$$

of the total energy. The reason why we prefer $Q$ rather than $H$ is that $Q$ is a nondecreasing interval function;

$$
\begin{aligned}
& \left|v_{i}\right| \leqq\left[2 Q\left(\omega, x_{i}, \sigma\right)\right]^{1 / 2}, \\
& U\left(x_{i}-x_{j}\right) \leqq Q\left(\omega, x_{i}, \sigma\right) \text { if }\left|x_{i}-x_{j}\right| \leqq \sigma,
\end{aligned}
$$

therefore any bound on $Q$ implies bounds on velocities and for interparticle distances as well. Let us remark that $Q$ is just the potential energy in the presence of a negative chemical potential $-B$.

The order of energy fluctuation at infinity (boundary condition in terms of Lanford [5]) is characterized by a moderately increasing (MI) function $g$.

Definition 1. A continuous even function $g=g(x)$ is called an MI-function if $g$ is increasing and concave for $x \geqq 0$ with right derivative $g^{\prime}(0+0)=1$; for convenience we assume that $g(0)=3 R+16(1+b)^{2}, b$ is given in (E) below. We say that the energy fluctuation of $\omega \in \Omega$ is only of order $g$ if

$$
\bar{Q}_{g}(\omega)=\sup _{m \in I} \sup _{\sigma \geqq g\left(x_{m}\right)} \frac{1}{2 \cdot \sigma} Q\left(\omega, x_{m}, \sigma\right)
$$

is finite, the set of such configurations will be denoted by $\bar{\Omega}_{g}$.

The particular cases $\bar{g}(x)=g(0)+|x|$ and $g_{0}(x)=g(0)+\log (1+|x|)$ are of special interest, the corresponding objects will be denoted by $\bar{Q}, \bar{\Omega}$ and $\bar{Q}_{0}, \bar{\Omega}_{0}$, respectively; $\log u$ denotes logarithm to base $e$. It is plain that $\bar{\Omega}_{g} \subset \bar{\Omega}_{h}$ if $g \leqq h$, thus $\bar{\Omega}_{g} \subset \bar{\Omega}$ for each MI-function $g$. Limiting solutions will be constructed for initial configurations 
$\omega \in \bar{\Omega}$ and we shall see that the order of energy fluctuation, i.e. the sets $\bar{\Omega}_{g}, \bar{\Omega}-\bar{\Omega}_{g}$, is an invariant of motion.

Let us remark that

$$
\bar{Q}_{g}(\omega) \leqq \sup _{\mu \in I} \sup _{\sigma \geqq g(|\mu|-1)} \frac{1}{2 \cdot \sigma} Q(\omega, \mu, 1+\sigma)
$$

for each MI-function $g$. Further, $Q(\omega, \mu, \sigma) \leqq Q(\omega, 0,|\mu|+\sigma)$ and $|\mu| \leqq \sigma$ in (7) if $g=\bar{g}$, thus the Ergodic theorem [13] implies that $\bar{\Omega}$ is of full measure with respect to any translation invariant field such that $H(\omega, 0, R)$ has a finite expectation. On the other hand, even $\bar{\Omega}_{0}$ carries a wide class of Gibbsian fields:

Proposition 1. If $\tilde{P}$ is the probability measure of a Gibbsian field associated with a finite-range pair potential $\tilde{U}$ such that

$$
U(x) \leqq \tilde{a}+\tilde{b} \tilde{U}(x)
$$

with some constants $\tilde{a}$ and $\tilde{b}$, then $\tilde{P}\left(\bar{\Omega}_{0}\right)=1$.

The proof of this statement is very simple because using (8) and taking into account that $U(x)=+\infty$ if $|x| \leqq \delta$, a direct calculation shows that

$$
\int_{\Omega} \exp (\lambda Q(\omega, \mu, \sigma)) P(d \omega) \leqq e^{2 c \sigma}
$$

with some $\lambda>0, c<+\infty$ that do not depend on $\mu$ and $\sigma$. Hence $P\left[Q(\omega, \mu, \sigma)>\frac{2 \sigma K}{\lambda}\right]$ $\leqq \exp [(c-K) 2 \sigma]$, thus Proposition 1 follows from (7) by the Borel-Cantelli lemma [13]. It seems that this proposition can be generalized e.g. for multibody potentials of infinite range of interaction, but condition (8) cannot be completely removed.

We can't disprove that even in the presence of limiting solutions there may exist further solutions oscillating rather violently at remote places of the line. Anyway, only such solutions are tractable where the order of energy fluctuations is limited not only at the initial moment of time, but later as well.

Definition 2. We say that a solution $\omega_{t}$ is $g$-tempered if $\bar{Q}_{g}\left(\omega_{t}\right)$ is bounded in finite intervals of time, in the particular case $g(x)=g(0)+|x|$ the prefix $g$ will be omitted.

In the proof of existence we need an additional regularity condition on the interaction: There exist such constants $a$ and $b$ that

$$
\left|(x-\delta) U^{\prime}(x)\right| \leqq a+b U(x) \text { if } x>\delta .
$$

Observe that (E) implies $U(x) \leqq a_{0}+b_{0}(x-\delta)^{-b}$ for $x>\delta$ and this relation is sufficient for $(\mathrm{E})$ if $U$ is convex in a small neighbourhood $(\delta, \delta+\varepsilon)$ of $\delta$. Although it is not everywhere necessary, the validity of $(\mathrm{E})$ will be assumed throughout this paper. The main result is

Theorem 1. For each $\omega \in \bar{\Omega}$ there exists a limiting solution $\omega_{t}$ with initial condition $\omega_{0}$ $=\omega$, and any limiting solution $\bar{\omega}_{t}$ is tempered if $\bar{\omega}_{0} \in \bar{\Omega}$.

On the structure of tempered solutions we prove the following.

Theorem 2. Let $g$ denote an MI-function, then a tempered solution $\omega_{t}$ is $g$-tempered if and only if $\omega_{0} \in \bar{\Omega}_{g}$, further if $\omega_{t} \in \bar{\Omega}_{g}$ at least for one value of $t>0$, then $\omega_{t} \in \bar{\Omega}_{g}$ for each $t \geqq 0$. 
Theorems 1 and 2 will be proved in Sections 5 and 6. Uniqueness of $g$-tempered solutions will be deduced from a quasi-Lipschitz condition of the following kind. We say that the right hand side of (1) satisfies a $g$-Lipschitz condition with an MIfunction $g$ if

$$
\lim _{n} n^{-2} L(v g(n))=0 \quad \text { for each } \quad v>0
$$

where $L=L(u)$ is the Lipschitz constant of $U^{\prime}$ in the domain $\{x ; U(x) \leqq u\}, L$ is defined before (1). The principal content of $(U)$ is that the singularity of $U$ is not very weak, i.e. the interparticle force is repulsive enough. For example, if $U^{\prime}$ is concave in a neighbourhood $(\delta, \delta+\varepsilon)$ of $\delta$ and $U(x) \geqq b_{1}(x-\delta)^{-c}$ in this interval, further $b_{1}>0$, $0<c \leqq 2$ and $0<\lambda \leqq \frac{2 c}{c+2}$, then (U) holds with $g_{\lambda}(x)=g(0)+\frac{1}{\lambda}\left[(1+|x|)^{\lambda}-1\right]$.

Theorem 3. Suppose that the right hand side of (1) satisfies a $g$-Lipschitz condition with an MI-function $g$. Then for each $\omega \in \bar{\Omega}_{g}$ there exists the limit $U_{t} \omega=\lim _{n} \varphi_{n}(t, \omega)$ of approximate solutions $\varphi_{n}$ in the topology of $\Omega[0, \infty) . U_{t}$ is a reversible semigroup of $g$-tempered solutions, and the restriction of $U_{t} \bar{\Omega}_{q} \mapsto \Omega[0, \infty)$ to $\bar{\Omega}_{g}^{q}=\left\{\omega ; \bar{Q}_{g}(\omega) \leqq q\right\}$ is a continuous function of $\omega \in \bar{\Omega}_{g}^{q}$. Further, $\omega_{t}=U_{t} \omega_{0}$ is the only tempered solution with initial condition $\omega_{0} \in \bar{\Omega}_{g}$.

The proof of this result will be given in Section 7 .

\section{On the Idea of the Proof}

The crucial step in the proof of Theorem 1 is to verify compactness of the sequence $\varphi_{n}$ of approximate solutions. For this we deduce an a priori bound $\bar{Q}\left(\varphi_{n}(t, \omega)\right) \leqq q(t, \omega)$ such that $q$ is independent of $n$ and continuous in $t \geqq 0$ for each $\omega \in \bar{\Omega}$. To explain on a heuristic level that how such a $q$ can be obtained, we replace $\bar{Q}$ by $\bar{Q}_{00}$ that corresponds to the constant function $g_{00}(x)=g(0)$. Although this situation is not very interesting because $\bar{Q}_{00}(\omega)=+\infty$ a.s. in nondegenerate Gibbsian fields, the general case is more difficult only in its technical aspects.

Consider $Q_{t}=Q\left[\varphi_{n}(t, \omega), x_{m}\left(\varphi_{n}(t, \omega)\right), r(t)\right]$ that is the quantity of $Q$ in the $r(t)$ neighbourhood $V_{t}$ of the $m$-th particle of $\varphi_{n}(t, \omega)$. In view of the law of energy conservation, the differential gain of $Q_{t}$ consists of two summands : (i) transported energy $Q_{t p}$ that amounts to the sum of chemical, kinetic and potential energy carried by particles crossing the boundary of $V_{t}$ and (ii) transferred energy $Q_{t f}$ that is the work of external forces on internal particles, this is a boundary effect, too. Let $0 \leqq t \leqq T, q_{n}(t, \omega)=\bar{Q}_{00}\left(\varphi_{n}(t, \omega)\right)$ and

$$
r(t)=\sigma+c \int_{t}^{T} \sqrt{q_{n}(s, \omega)} d s .
$$

Since all velocities in $\omega$ are bounded by $2 \sqrt{g(0) \bar{Q}_{00}(\omega)}$, for $c>2 \sqrt{g(0)}$ we have $-r^{\prime}(t) \geqq\left|v_{i}\left(\varphi_{n}(t, \omega)\right)\right|$, thus new particles cannot enter into $V_{t}$ so that $Q_{t p} \leqq 0$. One 
would expect that even $Q_{t p}+Q_{t f} \leqq 0$ holds if $c$ is large enough, i.e. $Q_{T} \leqq Q_{0}$, or more exactly,

$$
Q\left[\varphi_{n}(T, \omega), x_{m}\left(\varphi_{n}(t, \omega)\right), \sigma\right] \leqq Q_{0} \leqq Q\left[\omega, x_{m}(\omega), \sigma+c \int_{0}^{T} \sqrt{q_{n}(s, \omega)} d s\right]
$$

for each $T \geqq 0$. This heuristic argument remains in force in the $d$-dimensional space, too, where $V_{t}$ is the sphere of radius $r(t)$ and centre $x_{m}\left(\varphi_{n}(t, \omega)\right)$, further $\bar{Q}_{00}$ is the supremum of the quantities: $Q$ per volume; the volume is bounded away from zero in the definition of $\bar{Q}_{00}$. Dividing by the volume of $V_{t}$ and taking the supremum of both sides, (9) turns into

$$
q_{n}(T, \omega) \leqq \bar{Q}_{00}(\omega)\left[1+\bar{c} \int_{0}^{T} \sqrt{q_{n}(s, \omega)} d s\right]^{d},
$$

where $\bar{c}$ depends only on $c$ and $d$. It is easy to check that the maximal solution $q(t, \omega)$ of this integral inequality is bounded in finite intervals of time if $d \leqq 2$ and $\bar{Q}_{00}(\omega)<+\infty$, while $q(t, \omega)=+\infty$ for large values of $t$ if $d>2$, see Bihari's Inequality in $[10,11]$. Therefore we have a possibility to prove existence of solutions if $d=1$ or $d=2$, but such an argument does not work if $d \geqq 3$. Moreover, some examples indicate that (9) cannot be improved essentially, so that there is no hope for extending Theorem 1 in its deterministic form to three-dimensional particle systems.

Let us now turn to our main hypothesis $Q_{t p}+Q_{t f} \leqq 0$. Unfortunately this is not true in general because $Q_{t f}$ may be arbitrarily large if many particles are close to each other near the boundary of $V_{t}$. It is possible, however, to replace $Q$ by such an additive, energy-like quantity $W$ that the transport of $W$ overbalances its transfer even if particles interact very strongly near the boundary of $V_{t}$. For this our system will be transformed into a new one by removing the intervals occupied by the hard cores, the definition of $W$ for this new system will be essentially the same as that of $Q$ for the old one. Due to this contraction of the system, the formal transport of energy has been considerably enlarged in case of strongly interacting configurations so that we shall have $W_{t p}+W_{t f} \leqq 0$. In the absence of hard cores and in the two-dimensional space such a method does not work, some new ideas are needed then, see Section 8 and [9].

\section{The a priori Bound}

In this section we translate into mathematics the ideas outlined above. Let $f=f(x, \sigma)$ denote such a continuously differentiable version of the indicator $f_{0}$ of $[-\sigma, \sigma]$ that the partial derivatives $f_{1}=\frac{\partial f}{\partial x}$ and $f_{2}=\frac{\partial f}{\partial \sigma}$ are continuous in the domain $-\infty<x<+\infty, \sigma>0$, further

(i) $0 \leqq f(x, \sigma) \leqq 1$ for each $x$ and $\sigma>0$,

(ii) $f(x, \sigma)=1$ if $|x| \leqq \sigma, f(x, \sigma)=0$ if $|x| \geqq \sigma+2 R$,

(iii) $\left|f_{1}(x, \sigma)\right| \leqq f_{2}(x, \sigma)$ for each $x$ and $\sigma>0$,

(iv) $\left|f_{1}(u, \sigma)\right| \leqq f_{2}(x, \sigma)+f_{2}(y, \sigma)$ if $x<u<y \leqq x+R$. 
For example if $e(x)$ is a continuously differentiable nonincreasing function such that $e(x)=1$ if $x \leqq 0, e(x)=0$ if $x \geqq 2 R, e(x)$ is concave for $x \leqq 3 \frac{R}{2}$ and convex for $x \geqq \frac{R}{2}$, i.e. it is linear if $|x-R| \leqq \frac{R}{2}$, then $f(x, \sigma)=e(|x|-\sigma)$ satisfies (i)-(iv). Now we introduce a smooth and additive version $W$ of $Q$ satisfying our hypothesis on the balance of transport and transfer. For notational convenience the reduced positions $y_{i}=y_{i}(\omega)=x_{i}(\omega)-i \delta$ will be used simultaneously with the original co-ordinates $x_{i}=x_{i}(\omega) . W$ is defined as a function of $\omega \in \Omega, \sigma>0$ and $m \in I$ by

$$
W_{m}(\omega, \sigma)=\sum_{i \in I} f\left(y_{i}-y_{m}, \sigma\right)\left[2 B+v_{i}^{2}+\sum_{j \neq i} U\left(x_{i}-x_{j}\right)\right] .
$$

Let us remember that the constant $B=\frac{a R}{b \delta}$ is so large that each term on the right hand side of (11) is nonnegative, thus $W_{m}$ is a nondecreasing function of $\sigma$, and

$$
\frac{\partial}{\partial \sigma} W_{m}(\omega, \sigma)=\sum_{i \in I} f_{2}\left(y_{i}-y_{m}, \sigma\right)\left[2 B+v_{i}^{2}+\sum_{j \neq i} U\left(x_{i}-x_{j}\right)\right]
$$

is also nonnegative. Let $\omega_{t}$ denote a solution of (1), then differentiating $W$ with respect to $t$, and exploiting $U^{\prime}(x)=-U^{\prime}(-x)$ and the equations of motion, an easy calculation yields

$$
\begin{aligned}
\frac{\partial}{\partial t} W_{m}\left(\omega_{t}, \sigma\right)= & \sum_{i \in I}\left[\frac{\partial}{\partial t} f\left(y_{i}-y_{m}, \sigma\right)\right]\left[2 B+v_{i}^{2}+\sum_{j \neq i} U\left(x_{i}-x_{j}\right)\right] \\
& +\frac{1}{2} \sum_{i \in I} \sum_{j \neq j}\left[f\left(y_{j}-y_{m}, \sigma\right)-f\left(y_{i}-y_{m}, \sigma\right)\right]\left(v_{i}+v_{j}\right) U^{\prime}\left(x_{i}-x_{j}\right) .
\end{aligned}
$$

It is very important that the sequence $\varphi_{n}$ of approximate solutions has been constructed in such a way that (13) holds even if $\omega_{t}=\varphi_{n}(t, \omega)$. In case of a finite system we can put $f=1$ for each $x$, then (13) reduces to $\frac{\partial W}{\partial t}=0$ that is the law of energy conservation.

Let us remark that the first and second sum in (13), respectively, are just the intensity of transport and transfer of $W$, further $V \frac{\partial}{\partial \sigma} W_{m}\left(\omega_{t}, \sigma\right)$ is the intensity of transport that results from compressing $\left[x_{m}-\sigma, x_{m}+\sigma\right]$ with velocity $V$. Therefore Lemma 1 describes how transport overbalances transfer.

Lemma 1. Let $\omega_{t}$ denote either a solution or an approximate solution $\varphi_{n}\left(t, \omega_{0}\right)$ defined in (2), and suppose that we are given such a bound $V$ that

$$
\left|\frac{d}{d t} x_{i}\left(\omega_{t}\right)\right| \leqq V \quad \text { if } \quad\left|y_{i}-y_{m}\right| \leqq \sigma+3 R
$$

holds for some $m, t, \sigma$; then

$$
\frac{\partial}{\partial t} W_{m}\left(\omega_{t}, \sigma\right) \leqq 2(b+1) V \frac{\partial}{\partial \sigma} W_{m}\left(\omega_{t}, \sigma\right) .
$$


Proof. Using property (iii) to estimate the first sum in (13) and (iv) for the second one we obtain that

$$
\begin{aligned}
\frac{\partial}{\partial t} W_{m}\left(\omega_{t}, \sigma\right) \leqq & \cdot V \frac{\partial}{\partial \sigma} W_{m}\left(\omega_{t}, \sigma\right) \\
& +V \sum_{i \in I} \sum_{j \neq i}\left[f_{2}\left(y_{j}-y_{m}, \sigma\right)+f_{2}\left(y_{i}-y_{m}, \sigma\right)\right]\left|\left(y_{j}-y_{i}\right) U^{\prime}\left(x_{i}-x_{j}\right)\right| \\
= & 2 V\left[\frac{\partial}{\partial \sigma} W_{m}+\sum_{i \in I} f_{2}\left(y_{i}-y_{m}, \sigma\right) \sum_{j \neq i}\left|y_{i}-y_{j}\right|\left|U^{\prime}\left(x_{i}-x_{j}\right)\right|\right] \\
\leqq & 2 V\left[\frac{\partial}{\partial \sigma} W_{m}+\sum_{i \in I} f_{2}\left(y_{i}-y_{m}, \sigma\right)\left[\frac{2 a R}{\delta}+b \sum_{j \neq i} U\left(x_{i}-x_{j}\right)\right]\right]
\end{aligned}
$$

at the last step (E) and $\left|y_{i}-y_{j}\right| \leqq\left|x_{i}-x_{j}\right|-\delta$ were used. Since $B=\frac{a R}{b \delta}$, this inequality proves the statement.

The next step is to show that fluctuations of $W$ are of the same order as that of $Q$. Set

$$
\bar{W}_{g}(\omega)=\sup _{m \in I} \sup _{\sigma \geqq g\left(x_{m}\right)} \frac{1}{2 \cdot \sigma} W_{m}(\omega, \sigma)
$$

for each MI-function $g$ and $\omega \in \Omega$; if $g(x)=g(0)+|x|$ then the simplified notation $\bar{W}(\omega)$ will be used. It is plain that $2 \bar{Q}_{g}(\omega) \leqq \bar{W}_{g}(\omega)$, i.e. $\bar{W}_{g}(\omega)<\infty$ implies $\bar{Q}_{g}(\omega)<\infty$; the converse statement is less trivial.

Lemma 2. There exists an increasing function $w=w(q), q \geqq 0$ depending only on $U$ such that $\bar{Q}_{g}(\omega) \leqq q<\infty$ implies $\bar{W}_{g}(\omega) \leqq w(q)<\infty$.

Proof. Let $\sigma \geqq g\left(x_{m}\right)$ and

$$
\varrho=\max _{i}\left\{\left|x_{i}-x_{m}\right| ;\left|y_{i}-y_{m}\right| \leqq \sigma+2 R\right\},
$$

then $W_{m}(\omega, \sigma) \leqq 2 Q\left(\omega, x_{m}, \varrho\right)$, thus

$$
\frac{1}{2 \cdot \sigma} W_{m}(\omega, \sigma) \leqq 2 q \max \left\{1, \frac{\varrho}{\sigma}\right\} \text {. }
$$

We may assume without any loss of generality that $\varrho \geqq 2 \sigma+4 R$ and the maximum in (15) is attained at $i=m+k, k>0$, then $\varrho \leqq \sigma+2 R+k \delta$, whence $\varrho \leqq 2 k \delta$ follows directly, thus from (D) we obtain that

$$
q \geqq \frac{1}{2 \cdot \varrho} Q\left(\omega, x_{m}, \varrho\right) \geqq \frac{1}{4 k \delta} \sum_{i=m+1}^{m+k} U\left(x_{i}-x_{i-1}\right) .
$$

On the other hand, let $V=V(x), x \geqq 0$ denote the supremum of all convex functions $h=h(x), x \geqq 0$ such that $h(x) \leqq U(x)$ if $x \geqq 0$. As a supremum of convex functions, $V$ is convex again for $x>\delta$, and $V(\delta+0)=+\infty$ as $U(\delta+0)=+\infty$. Thus by Jensen's Inequality we have $4 \delta q \geqq V\left(\frac{\varrho}{k}\right)$ as $\varrho=x_{m+k}-x_{m}$, and this is possible only if there exists an $\varepsilon=\varepsilon(q)>0$ such that $\varrho \geqq k(\delta+\varepsilon)$. Since $\varrho \leqq \sigma+2 R+k \delta$, it follows that 
$k \leqq \frac{1}{\varepsilon}(\sigma+2 R)$, i.e. $\varrho \leqq\left(1+\frac{\delta}{\varepsilon}\right)(\sigma+2 R)$. Therefore $\frac{\varrho}{\sigma}$ remains bounded if $q$ is fixed, thus (16) implies the statement.

We are now in a position to deduce the a priori bound implying existence of solutions.

Proposition 2. Let $\omega_{t}$ denote either a g-tempered solution of (1) or an approximate solution $\omega_{t}=\varphi_{n}\left(t, \omega_{0}\right)$, and suppose that $\bar{Q}_{g}\left(\omega_{0}\right) \leqq q<+\infty ; g$ is an arbitrary MIfunction. Then

$$
\bar{Q}_{g}\left(\omega_{t}\right) \leqq w(q) \exp (2 t \sqrt{w(q)}) \text { for } t \geqq 0
$$

with $w=w(q)$ as specified in Lemma 2. Further

$$
\sigma_{m}\left(\omega_{t}\right)=\max _{0 \leqq s \leqq t}\left|x_{m}\left(\omega_{s}\right)-x_{m}\left(\omega_{0}\right)\right|
$$

satisfies the inequality

$$
\left[g\left(\left|x_{m}\left(\omega_{0}\right)\right|+\sigma_{m}\left(\omega_{t}\right)\right)\right]^{1 / 2} \leqq\left[g\left(x_{m}\left(\omega_{0}\right)\right]^{1 / 2}+g^{\prime}\left(\left|x_{m}\left(\omega_{0}\right)\right|+0\right)\left(e^{t \sqrt{w(q)}}-1\right)\right.
$$

for each $m$ and $t \geqq 0 ; g^{\prime}(u+0)$ denotes the right hand side derivative of $g$.

Proof. Observe first that in view of $v_{i}^{2} \leqq W_{i}\left(\omega_{t}, R\right)$,

$$
V=V_{m}\left(\omega_{t}, \sigma\right)=\left[2 \bar{W}_{g}\left(\omega_{t}\right) g\left(\left|x_{m}\left(\omega_{t}\right)\right|+\sigma+3 R\right)\right]^{1 / 2}
$$

satisfies the requirement of Lemma 1 . Further, any approximate solution is a $g$ tempered one, i.e. $\bar{W}_{g}\left(\omega_{t}\right)$ is bounded in finite intervals of time; consequently the integral equation

$$
r(t)=\sigma+2(b+1) \int_{t}^{T} V_{m}\left(\omega_{s}, r(s)\right) d s, \quad 0 \leqq t \leqq T,
$$

has a unique solution $r=r(t)$ for each $\sigma>0, T>0$ such that $r(T)=\sigma$ and $r(t)$ decreases so rapidly that the partial differential inequality of Lemma 1 turns into $\frac{d}{d t} W_{m}\left(\omega_{t}, r(t)\right) \leqq 0$, i.e.

$$
W_{m}\left(\omega_{T}, \sigma\right) \leqq W_{m}\left(\omega_{0}, r(0)\right) .
$$

Suppose now that $\sigma \geqq g\left(x_{m}\left(\omega_{T}\right)\right)$; since $\left|\frac{d}{d t} x_{m}\left(\omega_{t}\right)\right| \leqq-r^{\prime}(t)$,

$$
\left|x_{m}\left(\omega_{t}\right)\right| \leqq\left|x_{m}\left(\omega_{T}\right)\right|+r(t)-\sigma \quad \text { if } \quad 0 \leqq t \leqq T .
$$

On the other hand, any MI-function satisfies

$$
g(x+y) \leqq g(x)+g^{\prime}(x+0) y \leqq g(x)+y \quad \text { if } \quad x, y \geqq 0,
$$

whence $g\left(x_{m}\left(\omega_{0}\right)\right) \leqq g\left(x_{m}\left(\omega_{T}\right)\right)+r(0)-\sigma \leqq r(0)$. Therefore $W_{m}\left(\omega_{0}, r(0)\right) \leqq 2 r(0) \bar{W}_{g}\left(\omega_{0}\right)$ so that dividing by $2 \sigma$ and taking the supremum of both sides in (18) we get

$$
\bar{W}_{g}\left(\omega_{T}\right) \leqq \bar{W}_{g}\left(\omega_{0}\right) \sup _{\sigma} \frac{r(0)}{\sigma},
$$

where $r(0)$ and $\sigma$ are related by (17). 
From (19), (20) and $r(t) \leqq r(0)$ we obtain that

$$
\begin{aligned}
g\left(\left|x_{m}\left(\omega_{t}\right)\right|+r(t)+3 R\right) & \leqq g\left(x_{m}\left(\omega_{T}\right)\right)+2 r(0)-\sigma+3 R \\
& \leqq 2 r(0)+3 R \text { if } 0 \leqq t \leqq T,
\end{aligned}
$$

thus (17) implies that

$$
r(0) \leqq \sigma+4(b+1) u(T) \sqrt{r(0)+\frac{3}{2} R},
$$

where

$$
u(t)=\int_{0}^{t} \sqrt{\bar{W}_{g}\left(\omega_{s}\right)} d s .
$$

Since $r(0) \geqq \sigma \geqq g(0)=3 R+16(b+1)^{2}$, we have

$$
\sqrt{r(0)} \leqq \sqrt{\sigma}+4(b+1) u(T)\left[1+\frac{3 R}{2 g(0)}\right]^{1 / 2},
$$

whence

$$
\sqrt{\frac{r(0)}{\sigma}} \leqq 1+u(T)
$$

follows by an easy calculation. Therefore (21) turns into

$$
\bar{W}_{g}\left(\omega_{t}\right) \leqq \bar{W}_{g}\left(\omega_{0}\right)\left[1+\int_{0}^{t} \sqrt{\bar{W}_{g}\left(\omega_{s}\right)} d s\right]^{2}, \quad t \geqq 0,
$$

where $\bar{W}_{g}\left(\omega_{0}\right) \leqq w(q)$ by Lemma 2 . This means that $v(t)=\sqrt{\bar{W}_{g}\left(\omega_{t}\right)}$ satisfies the integral inequality

$$
v(t) \leqq \sqrt{w(q)}\left(1+\int_{0}^{t} v(s) d s\right)
$$

for each $t \geqq 0$. The maximal solution of this inequality is just $q(t)$

$=\sqrt{w(q)} \exp (t \sqrt{w(q)})$ (see a variant of Gronvall's lemma in Section 4.4 of [10]), thus (Q) follows from the trivial relation $\bar{Q}_{g}(\omega) \leqq \bar{W}_{g}(\omega)$.

To prove $(\mathrm{X})$ observe that $(\mathrm{V})$ implies

$$
\left|x_{m}\left(\omega_{t}\right)-x_{m}\left(\omega_{0}\right)\right| \leqq \int_{0}^{t}\left[4 \bar{Q}_{g}\left(\omega_{s}\right) g\left(x_{m}\left(\omega_{s}\right)\right)\right]^{1 / 2} d s
$$

thus $\sigma_{m}\left(\omega_{t}\right)$ satisfies

$$
\sigma_{m}\left(\omega_{t}\right) \leqq 2 \int_{0}^{t}\left[\bar{Q}_{g}\left(\omega_{s}\right) g\left(\left|x_{m}\left(\omega_{0}\right)\right|+\sigma_{m}\left(\omega_{s}\right)\right]^{1 / 2} d s,\right.
$$

whence by Bihari's inequality (see [11] or Section 4.5. in [10]) and by (Q) we obtain that

$$
\int_{0}^{\sigma_{m}\left(\omega_{t}\right)}\left[g\left(\left|x_{m}\left(\omega_{0}\right)\right|+u\right)\right]^{-1 / 2} d u \leqq 2 \int_{0}^{t}\left[\bar{Q}_{g}\left(\omega_{s}\right)\right]^{1 / 2} d s \leqq 2\left(e^{t \sqrt{w(q)}}-1\right) .
$$


Multiplying this inequality by $g^{\prime}\left(\left|x_{m}\left(\omega_{0}\right)\right|+0\right)$ and taking into account that $0<g^{\prime}\left(\left|x_{m}\left(\omega_{0}\right)\right|+u+0\right) \leqq g^{\prime}\left(\left|x_{m}\left(\omega_{0}\right)\right|+0\right)$ if $u \geqq 0$ we get

$$
\begin{aligned}
& \int_{0}^{\sigma_{m}\left(\omega_{t}\right)} \frac{1}{2} g^{\prime}\left(\left|x_{m}\left(\omega_{0}\right)\right|+u+0\right)\left[g\left(\left|x_{m}\left(\omega_{0}\right)\right|+u\right)\right]^{-1 / 2} d u \\
& \leqq g^{\prime}\left(\left|x_{m}\left(\omega_{0}\right)\right|+0\right)\left(e^{t \sqrt{w(q)}}-1\right) ;
\end{aligned}
$$

the integral on the left can be calculated explicitely, the result is just (X).

In the proof of uniqueness of tempered solutions the following assertion will be needed.

Proposition 3. A tempered solution $\omega_{t}$ is $g$-tempered with an MI-function $g$ if and only if $\omega_{0} \in \bar{\Omega}_{g}$.

Proof. $g(x) \leqq g(0)+|x|$ implies that any $g$-tempered solution is tempered. Let $\omega_{t}$ denote a tempered solution with $\bar{Q}_{g}\left(\omega_{0}\right)=q<+\infty$ and define the sequence $g_{n}$, $n=1,2, \ldots$ of even functions by $g_{n}(x)=g(x)$ if $|x| \leqq n-1$, $g_{n}(x)=g(n-1)+g^{\prime}(n-1+0)(|x|-n+1)$ if $|x|>n-1$; each $g_{n}$ is an MI-function such that $g(x) \leqq g_{n}(x)$, whence $\bar{Q}_{g_{n}}\left(\omega_{0}\right) \leqq q$ follows by the definition of $\bar{Q}$. On the other hand, for each $n$ there exists a constant $\varrho_{n}<\infty$ such that $g(0)+|x| \leqq \varrho_{n} g_{n}(x)$, thus the monotonicity of $Q$ as a function of $\sigma$ implies that $\bar{Q}_{g_{n}}\left(\omega_{t}\right) \leqq \varrho_{n} \bar{Q}\left(\omega_{t}\right)$ for each $n$ and $t \geqq 0$; i.e. $\omega_{t}$ is $g_{n}$-tempered. Therefore (Q) of Proposition 2 yields

$$
\frac{1}{2 \cdot \sigma} Q\left(\omega_{t}, x_{m}\left(\omega_{t}\right), \sigma\right) \leqq \bar{Q}_{g_{n}}\left(\omega_{t}\right) \leqq w(q) \exp (2 t \sqrt{w(q)})
$$

for $t \geqq 0, m \in I$ and $\sigma \geqq g_{n}\left(x_{m}\left(\omega_{t}\right)\right)$. Since $Q(\omega, \mu, \sigma)=\inf _{\varrho>\sigma} Q(\omega, \mu, \varrho)$ and $g(x)=\inf _{n}$ $g_{n}(x),(26)$ holds even if $\sigma \geqq g\left(x_{m}\left(\omega_{t}\right)\right)$, which proves the statement.

\section{Proof of Existence}

Let us rewrite (1) into the concise form

$$
\frac{d}{d t} v_{i}\left(\omega_{t}\right)=F_{i}\left(\omega_{t}\right), \quad \frac{d}{d t} x_{i}\left(\omega_{t}\right)=v_{i}\left(\omega_{t}\right), \quad i \in I,
$$

where

$$
F_{i}(\omega)=-\sum_{j \neq i} U^{\prime}\left[x_{i}(\omega)-x_{j}(\omega)\right]
$$

and observe that $(\mathrm{Q})$ and $(\mathrm{X})$ yield such a bound

$$
Q\left(\varphi_{n}(t, \omega), x_{i}\left(\varphi_{n}(t, \omega)\right), R\right) \leqq q_{i}(t, \omega), \omega \in \bar{\Omega}, t \geqq 0
$$

that $q_{i}$ is a continuous function of $t$ and does not depend on $n$. Hence it follows by (V) that the sequence $\varphi_{n}(t, \omega)$ of approximate solutions contains a subsequence converging in the topology of $\Omega[0, \infty)$ for each fixed initial configuration $\omega \in \bar{\Omega}$. Indeed, the Arzela-Ascoli theorem on the compactness of sets of continuous functions implies that for each $\omega \in \bar{\Omega}, i \in I$ and $T>0$ there exists such a subsequence 
$n_{k}$ that $x_{i}\left(\varphi_{n_{k}}(t, \omega)\right)$ converges uniformly in $[0, T]$. Applying the diagonal method first for $T_{n}=n$ with $\omega$ and $i$ fixed, and then for $i \in I$ with $\omega$ fixed, we can select a subsequence $\bar{n}_{k}$ such that each co-ordinate $x_{i}\left(\varphi_{\bar{n}_{k}}(t, \omega)\right)$ converges uniformly in any of the intervals $[0, T], T>0$.

On the other hand, (26) and (D) show that interparticle distances are bounded away from $\delta$, thus the approximate solutions remain in such a domain of $\Omega$ where each $F_{i}$ is continuous. Therefore substituting into

$$
v_{i}\left(\omega_{t}\right)=v_{i}\left(\omega_{0}\right)+\int_{0}^{t} F_{i}\left(\omega_{s}\right) d s
$$

we obtain that also $v_{i}\left(\varphi_{\bar{n}_{k}}(t, \omega)\right)$ converges uniformly in finite intervals of time for each $i$; i.e. $\varphi_{\bar{n}_{k}}(t, \omega)$ converges in the topology of $\Omega[0, \infty)$. Of course, $\bar{n}_{k}$ may depend on $\omega$. Finally, a similar argument shows that the above limit is a solution; $g$ temperedness of limiting solutions with initial data $\omega_{0} \in \bar{\Omega}_{g}$ is a direct consequence of the lower semicontinuity of $\bar{Q}_{g}(\omega)$ as a function of $\omega$, cf. $(\mathrm{Q})$. Thus Theorem 1 is proven.

Proof of Theorem 2. The first part of Theorem 2 is just Proposition 3. To prove the second one assume that $\omega_{t}$ is a tempered solution and $\omega_{t} \in \bar{\Omega}_{g}$ for $t=s>0$; we have to show that $\omega_{0} \in \bar{\Omega}_{g}$. Indeed, $\bar{\omega}_{t}=\omega_{s-t}^{+}$is a solution for $0 \leqq t \leqq s$, and $\bar{\omega}_{s}=\omega_{0}^{+} \in \bar{\Omega}$, thus in view of (27) and of Theorem 1, $\bar{\omega}_{t}$ can be continued as a tempered solution for $t>s$, too. Further, $\bar{\omega}_{0}=\omega_{s}^{+} \in \bar{\Omega}_{g}$, whence $\bar{\omega}_{s} \in \bar{\Omega}_{g}$ follows by Proposition 3, consequently $\omega_{0}=\bar{\omega}_{s}^{+}$also belongs to $\bar{\Omega}_{g}$.

\section{Dependence of Solutions on Initial Data}

In this section we derive a bound for the deviation of $g$-tempered solutions from each other in terms of their initial deviation. Let $\lambda_{n}$ denote an increasing sequence of positive numbers and define $d_{n}(\omega, \bar{\omega})$ as

$$
d_{n}(\omega, \bar{\omega})=\sum_{i \in I} f\left(x_{i}, 3 R n\right) f\left(\bar{x}_{i}, 3 R n\right)\left[\lambda_{n}\left|x_{i}-\bar{x}_{i}\right|+\left|v_{i}-\bar{v}_{i}\right|\right],
$$

where $x_{i}, v_{i}, \bar{x}_{i}, \bar{v}_{i}$ denote the corresponding positions and velocities of the $i$-th particle in the configurations $\omega$ and $\bar{\omega}$, while $f=f(x, \sigma)$ is the function described in Section 5 by (i)-(iv). We may assume that $f$ satisfies the additional condition that

$$
\left|f_{1}(x, \sigma)\right| \leqq K<+\infty \quad \text { for each } x \text { and } \sigma>0 .
$$

Of course, $d_{n}$ depends on the choice of correspondence for particles from $\omega$ and $\bar{\omega}$. (V) implies a trivial bound for $d_{n}$, namely if $\max \left\{\bar{Q}_{g}(\omega), \bar{Q}_{g}(\bar{\omega})\right\} \leqq q<+\infty$, then an easy calculation results in

$$
d_{n}(\omega, \bar{\omega}) \leqq \frac{1}{\delta}(\delta+6 R n+4 R)\left[\lambda_{n}(6 R n+4 R)+2 \sqrt{2 g(3 R n+2 R) q}\right] .
$$

A measure $D_{p}(\omega, \bar{\omega})$ for the deviation of $\omega$ and $\bar{\omega}$ from each other can be defined as follows; $p$ is an arbitrary positive number, $M_{n}=\lambda_{1} \lambda_{2} \ldots \lambda_{n-1}$ if $n>1, M_{1}=1$, and 
the sequence $\lambda_{n}$ will be specified in such a way that $\lim n^{-1} \lambda_{n}=0$, i.e. $\lambda_{n}=o(n)$. Then (29) implies that

$$
D_{p}(\omega, \bar{\omega})=\sum_{n=1}^{\infty} \frac{p^{n}}{n !} M_{n} d_{n}(\omega, \bar{\omega}), \omega, \bar{\omega} \in \bar{\Omega}
$$

is finite, and if $\omega_{n}$ and $\omega$ belong to $\bar{\Omega}$ then $\lim \omega_{n}=\omega$ holds in the weak topology of $\Omega$ if and only if $\lim _{n} D_{p}\left(\omega_{n}, \omega\right)=0$ with some $p>0$.

Proposition 4. Suppose that the right hand side of (1) satisfies a g-Lipschitz condition (U) with an MI-function $g$. Then for each $T>0$ and $q<+\infty$ there exist a sequence $\lambda_{n}=\lambda_{n}(q, T)=o(n)$ and a positive constant $p=p(q, T)$ such that

$$
\sup _{0 \leqq t \leqq T} D_{1}\left(\omega_{t}, \bar{\omega}_{t}\right) \leqq D_{p}\left(\omega_{0}, \bar{\omega}_{0}\right)
$$

holds for any couple $\omega_{t}, \bar{\omega}_{t}$ of g-tempered solutions satisfying $\max \left\{Q_{g}\left(\omega_{0}\right), Q_{g}\left(\bar{\omega}_{0}\right)\right\}$ $\leqq q$.

Proof. Let $q(t)=w(q) \exp (2 t \sqrt{w(q)})$ and suppose that $\max \left\{\left|x_{i}\left(\omega_{t}\right)\right|,\left|x_{i}\left(\bar{\omega}_{t}\right)\right|\right\}$ $\leqq(3 n+2) R$, then in view of $(\mathrm{Q})$ of Proposition 2 we have

$$
\begin{aligned}
& \left|U^{\prime}\left[x_{i}\left(\omega_{t}\right)-x_{j}\left(\omega_{t}\right)\right]-U^{\prime}\left[x_{i}\left(\bar{\omega}_{t}\right)-x_{j}\left(\bar{\omega}_{t}\right)\right]\right| \\
& \quad \leqq L(2 q(t) g(3 R n+3 R))\left(\left|x_{i}\left(\omega_{t}\right)-x_{i}\left(\bar{\omega}_{t}\right)\right|+\left|x_{j}\left(\omega_{t}\right)-x_{j}\left(\bar{\omega}_{t}\right)\right|\right),
\end{aligned}
$$

where $L=L(u)$ is the Lipschitz-function defined before (1). Therefore if

$$
\lambda_{n}=\max \left\{[L(2 q(T) g(3 R n+3 R))]^{1 / 2},[g(3 R n+3 R)]^{1 / 2}\right\},
$$

then $\lambda_{n}=o(n)$ in view of $(\mathrm{U})$, and for $0 \leqq t \leqq T$,

$$
\left|F_{i}\left(\omega_{t}\right)-F_{i}\left(\bar{\omega}_{t}\right)\right| \leqq \frac{2}{\delta} R \lambda_{n}^{2} \sum\left|x_{j}\left(\omega_{t}\right)-x_{j}\left(\bar{\omega}_{t}\right)\right|
$$

where the sum is over such values of $j$ that $\left|x_{i}-x_{j}\right| \leqq R$ or $\left|\bar{x}_{i}-\bar{x}_{j}\right| \leqq R$. Observe now that $d_{n}(t)=d_{n}\left(\omega_{t}, \bar{\omega}_{t}\right)$ is an absolutely continuous function of $t$ and, using the properties (i), (ii), (v) of $f$, its derivative can be estimated almost everywhere as

$$
\begin{aligned}
d_{n}^{\prime}(t) \leqq & 2 K d_{n+1}(t)[2 q(t) g(3 R n+3 R)]^{1 / 2} \\
& +\sum_{i \in I} f\left(x_{i}, 3 R n\right) f\left(\bar{x}_{i}, 3 R n\right)\left[\lambda_{n}\left|v_{i}-\bar{v}_{i}\right|+\left|F_{i}\left(\omega_{t}\right)-F_{i}\left(\bar{\omega}_{t}\right)\right|\right] \\
\leqq & {\left[2 K[2 q(T) g(3 R n+3 R)]^{1 / 2}+\lambda_{n}\left(\frac{2}{\delta}\right)^{2} 2 R^{2}\right] d_{n+1}(t) } \\
\leqq & C \lambda_{n} d_{n+1}(t) \text { a.e. in }(0, T),
\end{aligned}
$$

where, because of $g(3 R n+3 R) \leqq \lambda_{n}^{2}, C$ is a positive finite constant depending on $T, q$, $R, K, \delta$, and $g$, but $C$ does not depend on $n$. Hence a.e. in $(0, T)$ we have

$$
\frac{M_{n}}{n !} d_{n}^{\prime}(s)(1+C t-C s)^{n} \leqq-\frac{M_{n+1}}{(n+1) !} d_{n+1}(s) \frac{d}{d s}(1+C t-C s)^{n+1}
$$


if $0 \leqq s \leqq t \leqq T$ so that

$$
\begin{aligned}
\sum_{n=1}^{\infty} & \frac{d}{d s}\left[\frac{M_{n}}{n !} d_{n}(s)(1+C t-C s)^{n}\right] \leqq-C d_{1}(s) \\
& +\sum_{n=1}^{\infty} \frac{M_{n}}{n !} d_{n}^{\prime}(s)(1+C t-C s)^{n} \\
& +\sum_{n=1}^{\infty} \frac{M_{n+1}}{(n+1) !} d_{n+1}(s) \frac{d}{d s}(1+C t-C s)^{n+1} \leqq 0
\end{aligned}
$$

for almost every $s$ in $(0, t)$. Therefore, integrating from 0 to $t$ we obtain that

$$
D_{1}\left(\omega_{t}, \bar{\omega}_{t}\right) \leqq \sum_{n=1}^{\infty} \frac{M_{n}}{n !}(1+C t)^{n} d_{n}\left(\omega_{0}, \bar{\omega}_{0}\right) \leqq D_{p}\left(\omega_{0}, \bar{\omega}_{0}\right)
$$

if $0 \leqq t \leqq T$ and $p=1+C T$, which proves the Proposition.

Proof of Theorem 3. Since $D_{p}(\omega, \bar{\omega})=0$ if and only if $\omega=\bar{\omega}$, Proposition 4 implies uniqueness of $g$-tempered solutions, whence uniqueness of tempered solutions follows by Proposition 3. On the other hand, any limiting solution $\omega_{t}$ is $g$-tempered if $\omega_{0}$ belongs to $\bar{\Omega}_{g}$, thus any subsequence of $\varphi_{n}(t, \omega)$ converges to the same limit, i.e. there exists the limit $U_{t} \omega=\lim \varphi_{n}(t, \omega)$ if $\omega \in \bar{\Omega}_{g}$. This relation implies that $U_{t}$ is a reversible semigroup in $\bar{\Omega}_{g}$, the continuity of $U_{t} \omega$ as a function of $\omega$ follows from the bound given in Proposition 4.

\section{Potentials without Hard Core}

Here we discuss briefly the case of $\delta=0$, a more detailed study of this problem will be given in [9]. The only modification of notation used in previous sections is that we put $B=0$ in the Definition (5) of $Q$. Let $\Omega_{g}$ denote the set of such configurations $\omega \in \bar{\Omega}_{g}$ that

$$
\bar{N}_{g}(\omega)=\sup _{m} \sup _{\sigma \geqq g\left(x_{m}(\omega)\right)} \frac{1}{2 \cdot \sigma} N\left(\omega, x_{m}(\omega), \sigma\right)<+\infty ;
$$

for superstable potentials $\bar{Q}_{g}(\omega)<+\infty$ implies that also $\bar{N}_{g}(\omega)<+\infty$, see [12] or [14]. In this definition $g$ is an MI-function, but only the particular case $g(x)=g_{0}(x)=g(0)+\log (1+|x|)$ will be discussed ; the corresponding symbols are $\Omega_{0}$ and $\bar{N}_{0}(\omega)$.

Theorem 4. For each $\omega \in \Omega_{0}$ there exists a limiting solution $\omega_{t}$ such that $\omega_{0}=\omega$.

Proof. The proof of Theorem 1 is repeated with some modifications. Let $\omega_{t}=\varphi_{n}(t, \omega)$ denote an approximate solution corresponding to $\omega \in \Omega_{0}$, instead of $W_{m}$ the quantity

$$
Z_{m}(\omega, \sigma)=W_{m}(\omega, \sigma)+\frac{a}{b} \sum_{i \in I} f\left(x_{i}-x_{m}, \sigma\right) \sum_{j \neq i} f\left(x_{j}-x_{m}, \sigma+3 R\right)
$$

will be used. Of course, $y_{i}(\omega)=x_{i}(\omega)$ as $\delta=0$ in this case, $a$ and $b$ denote the constants specified in (E), thus $Z_{m}$ is a nonnegative and nondecreasing function of $\sigma$. 
Further, $v_{i}^{2}(\omega) \leqq Z_{i}(\omega, \sigma)$ and $U\left(x_{i}(\omega)-x_{j}(\omega)\right) \leqq Z_{i}(\omega, \sigma)$ if $\sigma>R$, consequently an a priori bound of type $(\mathrm{Q})$ for

$$
\bar{Z}_{g}(\omega)=\sup _{m} \sup _{\sigma \geqq g\left(x_{m}(\omega)\right)} \sigma^{-2} Z_{m}(\omega, \sigma)
$$

is sufficient to deduce existence of limiting solutions.

Suppose now that for some $t, m$, and $\sigma$ we have a bound

$$
\left|\frac{d}{d t} x_{i}\left(\omega_{t}\right)\right| \leqq V \quad \text { if } \quad\left|x_{i}\left(\omega_{t}\right)-x_{m}\left(\omega_{t}\right)\right| \leqq \sigma+5 R ;
$$

then following the proof of Lemma 1 we obtain that

$$
\frac{\partial}{\partial t} Z_{m}\left(\omega_{t}, \sigma\right) \leqq 2(b+1) V \frac{\partial}{\partial \sigma} Z_{m}\left(\omega_{t}, \sigma\right) \text {. }
$$

Let $v(t)=\int_{0}^{t}\left[\bar{Z}_{g}\left(\omega_{s}\right)\right]^{1 / 2} d s, g$ is an MI-function. Since $\omega_{t}$ is an approximate solution, $v(t)$ and $v^{\prime}(t)$ are continuous functions. Set $\varrho(s)=\left|x_{m}\left(\omega_{s}\right)\right|+r(s)+5 R$ for $0 \leqq s \leqq T$, $T>0$ and observe that the solution $r(t), 0 \leqq t \leqq T$ of the integral equation

$$
r(t)=\sigma+2(b+1) \int_{t}^{T} g(\varrho(s)) v^{\prime}(s) d s
$$

decreases so rapidly that $\frac{d}{d t} Z_{m}\left(\omega_{t}, r(t)\right) \leqq 0$ if $0 \leqq t \leqq T$, thus $Z_{m}\left(\omega_{T}, \sigma\right) \leqq Z_{m}\left(\omega_{0}, r(0)\right)$, whence

$$
\bar{Z}_{g}\left(\omega_{T}\right) \leqq \bar{Z}_{g}\left(\omega_{0}\right) \sup _{\sigma}\left(\frac{r(0)}{\sigma}\right)^{2}
$$

follows as $\sigma \geqq g\left(x_{m}\left(\omega_{T}\right)\right)$ implies $r(0) \geqq g\left(x_{m}\left(\omega_{0}\right)\right)$ in the same way as in the proof of Proposition 1 .

From now on we specify $g$ as $g(x)=g_{0}(x)=g(0)+\log (1+|x|)$, the corresponding quantity $\bar{Z}_{g}(\omega)$ will be denoted by $\bar{Z}_{0}(\omega)$. Since we consider approximate solutions corresponding to the same initial value problem and this initial configuration $\omega$ belongs to $\Omega_{0}$, we have a finite constant $q=\left|\bar{Z}_{0}(\omega)\right|^{1 / 2}$ independent of $n$ in $\omega_{t}=\varphi_{n}(t, \omega),(43)$ turns into

$$
v^{\prime}(t) \leqq q \sup _{\sigma} \frac{r(0)}{\sigma} \text {. }
$$

On the other hand, $\varrho(s) \leqq\left|x_{m}\left(\omega_{T}\right)\right|+r(0)+5 R$ and $g_{0}(x+y) \leqq g_{0}(x)+g_{0}(y)$ if $x>0$, $y>0$, whence

$$
g_{0}(\varrho(s)) \leqq g_{0}\left(x_{m}\left(\omega_{T}\right)\right)+g_{0}(r(0)+5 R) \leqq \sigma+5 R+g_{0}(r(0)) \leqq 3 \sigma+g_{0}(r(0))
$$

follows directly, thus

$$
r(0) \leqq \sigma+2(b+1) v(T)\left(3 \sigma+g_{0}(r(0))\right) .
$$

Further, $\log r \leqq \sigma \log \frac{r}{\sigma}+\sigma$ if $r \geqq \sigma \geqq 1$ so that an easy calculation yields

$$
\frac{r(0)}{\sigma} \leqq 1+c v(T)\left(1+\log \frac{r(0)}{\sigma}\right) \leqq h(v(T)),
$$


where $c$ is a large enough constant depending only on $b$, while $h=h(v), v \geqq 0$ denotes the inverse function of the strictly increasing function $z(x)=\frac{x-1}{c+c \log x}, x \geqq 1$.
Consequently, (44) reduces to

$$
v^{\prime}(t) \leqq q h(v(t)),
$$

whence

$$
\int_{0}^{v(t)} \frac{1}{h(v)} d v \leqq q t
$$

Substituting $v=z(x)$ into this integral and taking into account that $\frac{1}{x \log x}$ is not integrable in $(1,+\infty)$, it follows immediately that the maximal solution of $(47)$ with initial condition $v(0)=0$ is bounded in finite intervals of time. This means that we have an a priori bound for $\bar{Z}_{0}\left(\varphi_{n}(t, \omega)\right)$ so that the proof can be completed in the same way as that of Theorem 1 .

\section{Concluding Remarks}

1. We can not construct the semigroup of motion without the quasi-Lipschitz condition. In this general case it is not known whether any tempered solution is a limiting one.

2. Estimating $\frac{r(0)}{\sigma}$ more carefully (e.g. as in the proof of Theorem 4) Proposition 2 can be improved. For example, if $g=g_{\lambda}(x)$ as defined before Theorem 3, then it follows that $\bar{Q}_{g}\left(\omega_{t}\right)$ increases only as fast as a power of $t$ depending on $\lambda<1$.

3. Theorem 4 holds for initial configurations satisfying $\bar{Z}_{0}(\omega)<+\infty$, this set is larger than $\Omega_{0}$. Similarily, if $\lambda>0$ and $\varepsilon>0$ are small enough, then Theorem 1 can be extended to such initial configurations for which

$$
\sup _{m} \sup _{\sigma \geqq g_{\lambda}\left(x_{m}(\omega)\right)} \sigma^{-1-\varepsilon} Q\left(\omega, x_{m}(\omega), \sigma\right)<+\infty
$$

4. Instead of $(E)$ it is enough to assume that

$$
\left|(x-\delta) U^{\prime}(x)\right| \leqq a+b U(x) \log (e+|U(x)|) .
$$

5. Conservation laws for the specific values of energy, particle number, impulse and entropy as well can be derived in a similar way as done by Lanford [2], Part II. Moreover, certain mixing properties of initial probability distributions are also preserved by the semigroup of motion.

\section{References}

1. Harris, T.E.: Diffusion with collision between particles. J. Appl. Prob. 2, 323-338 (1965)

2. Lanford,O.E.III.: The classical mechanics of one-dimensional systems of infinitely many particles.

I. An existence theorem. Commun. math. Phys. 9, 169-191 (1968)

II. Kinetic theory. Commun. math. Phys. 11, 257-292 (1968) 
3. Sinai, Ya.G.: Construction of the dynamics for one-dimensional systems of statistical mechanics. Teor. Mat. Fiz. 12, 487-497 (1972) (In Russian)

4. Sinai, Ya.G.: The construction of cluster dynamics for dynamical systems of statistical mechanics. Vestn. Moscow Univ. Ser. I. Mat. Mech. 29, 152-159 (1974) (In Russian)

5. Lanford,O.E.III.: Time evolution of large classical systems. In: Dynamical systems, theory and applications. Lecture notes in physics Vol. 38, pp. 1-111. Berlin-Heidelberg-New York: Springer 1975

6. Marchioro,C., Pellegrinotti, A., Presutti,E.: Existence of time evolution for $d$-dimensional statistical mechanics. Commun. math. Phys. 40, 175-185 (1975)

7. Presutti,E., Pulvirenti,M., Tirozzi, B. : Time evolution of infinite classical systems with singular, long range, two body interactions. Commun. math. Phys. 47, 81-95 (1976)

8. Lang,R.: Unendlich-dimensionale Wienerprozesse mit Wechselwirkung. Technical Report, Bielefeld Univ. (1976)

9. Dobrushin, R.L., Fritz,J.: Nonequilibrium dynamics of two-dimensional infinite particle systems. Technical Report, Mathematical Institute, Budapest (1977)

10. Beckenbach,E., Bellman, R.: Inequalities. In: Ergebnisse der Mathematik, Vol. 30. BerlinHeidelberg-Göttingen: Springer 1961

11. Bihari,I. : A generalization of a lemma of Bellman and its applications to uniqueness problems of differential equations. Ann. Math. Inst. Hungary 7, 81-94 (1956)

12. Ruelle,D.: Classical statistical mechanics of a system of particles. Helv. Phys. Acta 36, 183-197 (1963)

13. Loève,M.: Probability theory. Princeton-Toronto-New Jersey-London-New York: Van Nostrand 1955

14. Ruelle,D.: Statistical mechanics—rigorous results. New York-Amsterdam: Benjamin 1969

Communicated by J. L. Lebowitz

Received March 16, 1977 